\title{
ПОВЫШЕНИЕ ЧУВСТВИТЕЛЬНОСТИ АНАЛИЗА БЕНЗ(а)ПИРЕНА МЕТОДОМ ШПОЛЬСКОГО
}

\author{
(Представил О. Эйзен)
}

\section{1. Введение}

K настоящему времени как отечественными, так и зарубежными авторами разработан ряд методик количественного анализа бенз(а)пирена (БП) и других ароматических канцерогенных соединений $\left[{ }^{1-5}\right]$. Полный цикл анализа включает, как правило, четыре этапа [6]: 1) отбор пробы, 2) экстракцию, 3) разделение и 4) собственно анализ. Чувствительность анализа определяется главным образом его последним этапом, тогда как три предварительных лишь переводят анализируемое соединение без особых потерь в форму, удобную для детектирования. Наиболее чувствительным способом количественного измерения остается по сей день метод Шпольского, заключающийся в измерении интенсивностей линий в низкотемпературных $(T=77 \mathrm{~K})$ флуоресцентных спектрах анализируемых молекул, внедренных в качестве примесных в подходящие $н$-парафиновые матрицы [7] *.

Причины высокой чувствительности метода Шпольского просты. При низких температурах электронно-колебательные спектры поликристаллических твердых растворов состоят из квазилиний (полуширины при $77 \mathrm{~K}$ порядка $10-30 c \mu^{-1}$, при $4 \mathrm{~K}$ порядка $\left.1+10 c M^{-1}\right)$ и сопровождающих их фононных крыльев [8,9]. Нередко проявляется также неоднородность таких объектов в виде мультиплетов в смысле Шпольского [7]. Такое сужение спектра ведет, во-первых, к существенному повышению разрешающей способности $R=\lambda / \Delta \lambda$ (отношение положения линии к еe полуширине), что уменьшает вероятность случайного наложения на аналитические линии (по которым ведется количественная оценка) линий свечения постороннего происхождения. Во-вторых, на фоне с более широким спектральным распределением узкие линии можно измерить точнее, чем, к примеру, полосу свечения той же интегральной интенсивности (улучшается отношение сигнала к шуму). И, в-третьих, квазилинейчатость спектров поглощения приводит к принципиально новой возможности выделения полезного сигнала среди шума: можно селективно возбуждать флуоресценцию анализируемого соединения спектрально узкой полосой в резонансе с определенной линией поглощения.

По имеющимся сведениям, достигнут предел чувствительности по-

\footnotetext{
* Наряду с методом Шпольского для анализа применяются: 1) абсорбционная спектроскопия, 2) флуоресцентная спектроскопия при комнатной температуре, 3) цветовые и теплоцветовые тесты ['].
} 
рядка $10^{-10}$ г/мл БП** в измеряемом объеме замороженного раствора $H$-парафина $[1,10,11]$. При предельных измерениях равно важны два фактора: реальная чувствительность спектральной установки в комплекте с регистрирующей аппаратурой и достигаемая степень очистки реактивов, применяемых в процессе анализа. Кроме того, нельзя исключать и существования принципиального предела, связанного с конечным интервалом концентрации, где проявляется эффект Шпольского $\left[{ }^{12}\right]$.

В настоящей работе поставлена цель повысить чувствительность анализа БП методом Шпольского. Известно, что для накопления достаточного для анализа количества соединения часто нужны большие объемы первичных проб воды, воздуха, почвы и т. д. Их обработка, концентрирование, разделение, перевод в измерительный объем трудоемки, требуют значительных количеств специально очищенных реактивов. Повышение чувствительности (прежде всего абсолютной) самого анализа непосредственно связано с пропорциональным уменьшением объема проб, что приводит к экономии времени и средств. Кроме того, высокая чувствительность дает в руки исследователя тонкий инструмент для изучения распределения ароматических соединений, к примеру, в процессе их метаболизма в малых объектах, таких как небольшие участки пораженных опухолевыми процессами тканей, культуры, отдельные крупные клетки, в которые проник канцероген, и т. д.

\section{2. Операции с предельно разбавленными растворами БП}

Много усилий требует очистка $н$-парафинов и экстрагентов. Соответствующие методики, включающие ректификацию, химическую очистку и хроматографию, подробно описаны в [13]. Приготовить чистый $H$-парафин легче, чем сохранить его чистым. Трудности начинаются при концентрациях БП ниже $10^{-9}-10^{-10} \mu / \Omega$, что побудило принять ряд дополнительных мер.

А. Обеспечить чистоту лабораторной посуды, соприкасающейся с $\boldsymbol{H}$-парафинами, проведя цикл очистки в пять этапов. Первый - мытье посуды теплой водой и мылом. Второй - обычная сушка и обработка посуды химически чистым (без существенных следов люминесцирующих примесей) ацетоном для растворения следов органики. Третий обработка посуды в течение $2-3$ ч свежей хромовой смесью. Четвертый - многократное ополаскивание, сначала водопроводной, а затем дистиллированной водой. Пятый - сушка в чистом, предназначенном исключительно для этих работ сушильном шкафу без принудительной вентиляции. Чистая посуда хранилась под стеклянными колпаками. Б. Приспособить для разбавления растворов ниже $10^{-8}$ моль/ 6 П-НЖ из нержавеющей стали. Вытяжная вентиляция была заменена приточной и осуществлена обдувом из бытового пылесоса через последовательно соединенные колонну с активным углем и два пылеулавли вающих фильтра марки ФПП-00.

В. Открывать пустые запаянные медицинские ампулы (не нуждающиеся в дополнительной очистке) непосредственно в боксе, а раствор в них вводить по 1 мл с конуса стеклянной пипетки, после чего ампулы запаивать высоко над раствором быстрым нагреванием в пламени спиртовки.

Повышение чувствительности анализа неизбежно требует наличия контролируемого раствора БП с заданными концентрациями. Возмож-

Для БП $12 / \mu \Omega=4 \mu / \Omega$. 
ный предел разбавления может быть оценен после сопоставления данных методически верно проведенного цикла последовательных разбавлений с данными спектральных измерений и при констатации отсутствия в этом сопоставлении противоречий. Основой измерений служил концентрационный ряд (в $м / \Omega): 10^{-8}, 3 \cdot 10^{-9}, 10^{-9}$ и т. д. На исходном этапе небольшое количество БП растворялось в $\mu$-октане невысокой чистоты. После полного растворения (через сутки) раствор фотометрировался на «Specord» или «Acta MVII» относительно чистого $H$-октана. Начальная концентрация, составляющая величину порядка $10^{-5}$ (точность порядка $5 \%$ ), доводилась тремя этапами до $10^{-8} \mathrm{M} / \Omega$ (точность порядка $10 \%$ ). Каждый последующий этап разбавления увеличивал ошибку на $1-2 \%$.

Всегда существует реальный предел разбавления, определяемый 1) остаточной концентрацией БП в очищенном растворителе; 2) неконтролируемым загрязнением раствора БП на любом этапе разбавления (наиболее опасны конечные этапы); 3) фоном свечения любого происхождения на месте аналитической линии БП, не позволяющим судить о дальнейшем разбавлении; 4) пределом чувствительности регистрирующей системы. Последние два пункта не исключают дальнейшего разбавления, но отсутствие возможности контролировать такое разбавление с информационной точки зрения равносильно пределу разбавления. Имеется и принципиальный предел разбавления (когда флуктуации концентрации $\Delta N \gg N$ ), реализующийся при концентрациях $N<1$ молекулы в объеме измерения.

\section{3. Чувствительность регистрирующей системы}

Использована спектральная аппаратура, входящая в автоматизированный спектрометрический комплекс [14], обладающий всеми достоинст-
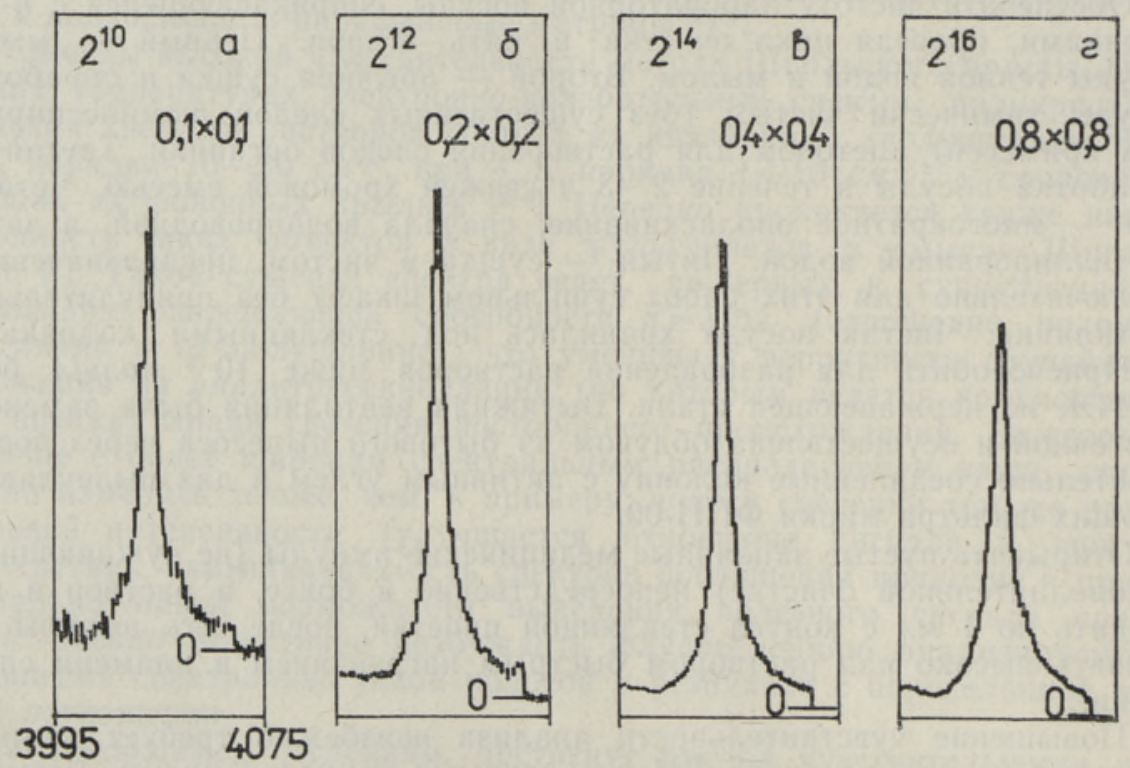

Рис, 1. Аналитическая $(0-0)$-линия БП $(4030 \AA)$ в н-октане при 77 К в объекте с концентрацией $3 \cdot 10^{-10} \mu / \Omega$ при разных щелях регистрации. Щели возбуждения $3 \times 3$ мм. 
Рис. 2. Общий вид барабанного объектодержателя в сосуде Дьюара.

вами, необходимыми для измерения сверхслабых свечений. В канале возбуждения применен двойной монохроматор МдР-1 (1:3), а в канале регистрации - ДФС-12 (1:5), на входной щели которого ФЭУ-79 с охлажденным примерно до $-30^{\circ} \mathrm{C}$ фотокатодом позволял измерять потоки фотонов вплоть до 3 фотонов в секунду на фотокатод при $4000 \AA$ А. На рис. 1 в качестве примера приведена аналитическая линия БП в объекте с концентрацией $3 \cdot 10^{-10}$ м/л при 77 К. Если учесть, что сигнал от такого объекта при максимальных щелях возбуждения $3 \times 3$ мм и щелях регистрации $0,8 \times 0,8$ мм составил $1,8 \cdot 10^{4}$ uмn/c, то, экстраполируя, можно получить аппаратурный предел чувствительности установки в режиме селективного возбуждения $10^{-14} \mathrm{M} / \Omega$ (или $\left.2,5 \cdot 10^{-15} 2 / M \Omega\right)$.

Для измерения концентрационных серий ампул с БП нами разработан и построен барабанный объектодержатель. Он вмещает до 20 ампул, погружается в жидкий азот и фиксирует положение ампул относительно перекрестия осей возбуждения и регистрации с точностью порядка $\pm 0,2 м м$ (рис. 2).

Рассмотрим далее вопрос выбора оптимального режима регистрации флуоресценции. В зависимости от характера спектра дополнительных примесей следует принять либо селективное, либо широкополосное возбуждение. Нельзя без уточнения ситуации говорить о преимуществе одного из вариантов перед другим. Вариант селективного возбуждения, при всех его достоинствах, обладает, как будет показано ниже, недостатком, выражающимся в усложнении спектра из-за эффектов типа комбинационного рассеяния (KP) и снятия неоднородного уширения $\left[{ }^{15}\right]$.

\section{4. Вариант селективного возбуждения}

Әтот вариант анализа позволяет во многих случаях проводить уверенное количественное определение БП в присутствии других люминесцирующих примесей. Изложим его особенности.

А. Положение полосы возбуждения выбирается в максимуме полосы поглощения БП в замороженном растворе. Мы проводили возбуждение на вибронной группе при $3884 \AA$, где молекула обладает наибольшим коэффициентом поглощения в ближней УФ-области. 

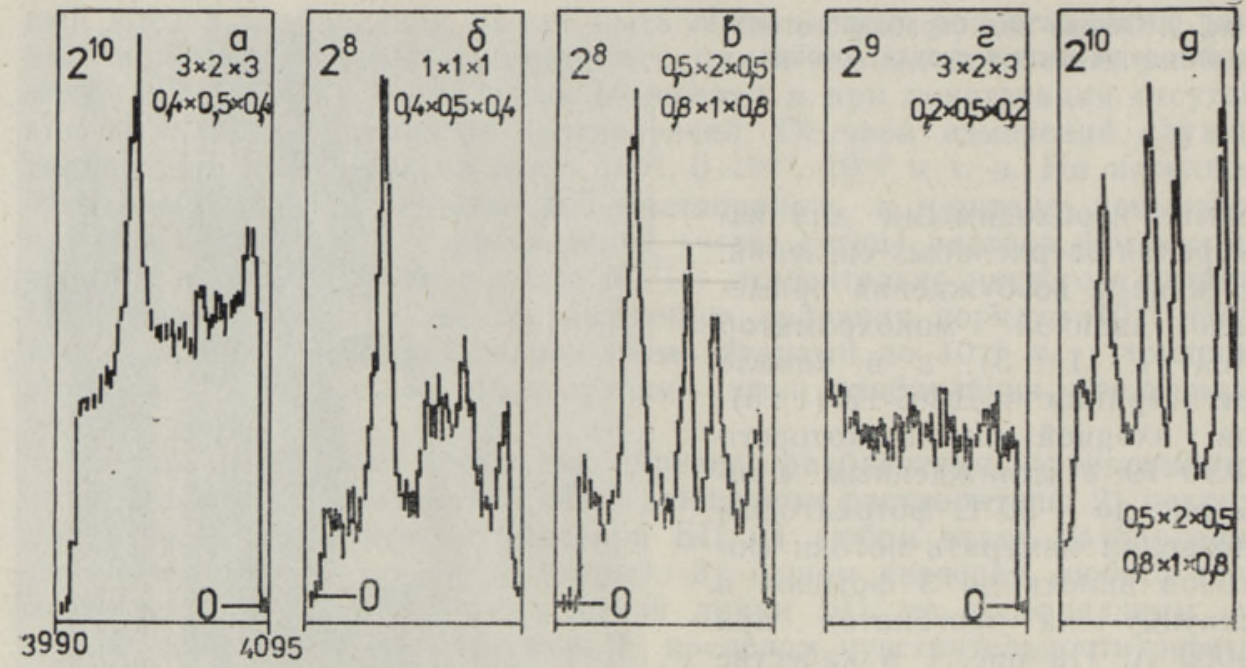

Рис. 3. $(0-0)$-линия БП в объекте с концентрацией $10^{-11}$ м/л при разных комбннациях щелей (приведено также значение средней щели) возбуждения и регистращии $(a-b)$. Тот же участок спектра, измеренный в объекте с чистым н-октаном $(2, \partial)$.

Б. Регистрация проводится на $(0-0)$-линии флуоресценции, наиболее интенсивной во всей серии и по этой причине принятой в качестве аналитической авторами всех методик анализа малых концентраций БП методом Шпольского. Для некоторого повышения селективности анализа, но ценой потери чувствительности, можно, в принципе, возбуждать в $(0-0)$-линии, а регистрировать на некотором вибронном повторении флуоресценции.

В. Оптимальная ширина полосы поглощения зависит от характера спектра примесей и должна отвечать двум противоречивым требованиям: 1) быть достаточно большой для большего значения интенсивности аналитической линии, 2) быть достаточно узкой, чтобы иметь лучшее отношение сигнала к шуму при регистрации сигнала от предельно разбавленных растворов. На рис. 3 в качестве примера показаны результаты измерения объекта с концентрацией БП $10^{-11} \mathrm{M} / \Omega$ при разных комбинациях щелей.

Г. Геометрия измерения стандартна, возбуждение и регистрация проводятся под углом $90^{\circ}$. Изображение выходной щели возбуждающего монохроматора фокусируется в виде полоски, размером примерно $10 \times 1-3$ мм, параллельно оси цилиндрической ампулы.

Д. Отметим еще один фактор принципиального характера, затрудняющий предельные измерения при селективном возбуждении. В актуальном для анализа участке спектра при выбранном положении и достаточной узости полосы возбуждения регистрируется участок спектра КР от основного вещества - матрицы н-октана (рис. 3,2 ). Первая полоса может быть отнесена к группе частот $842,860,877,895$ c ${ }^{-1}$; вторая $1028,1044,1062 \mathrm{~cm}^{-1}$; третья - 1085, $1137 \mathrm{~cm}^{-1}$; четвертая - $1304 \mathrm{~cm}^{-1}\left[{ }^{13}\right]$. В силу того, что $\boldsymbol{H}$-парафины обладают сложным спектром KР, при относительно малой энергетической разности положений возбуждения и регистрации $\left(<3000\right.$ c $\left.\boldsymbol{M}^{-1}\right)$ всегда существует опасность иметь на месте регистрации КР от матрицы.

На рис. 4 показаны результаты измерения спектров нижнего конца концентрационного ряда БП в н-октане. 




Рис. 4. $\left(0-0^{0}\right)$-линия БП в объектах с концентрациями $3 \cdot 10^{-10}(a), 10^{-10}(6)$, $3 \cdot 10^{-11}(8), 10^{-11} м / \Omega$ (2) при щелях возбуждения $1 \times 1 \times 1$. м. и $0,4 \times 0,5 \times 0,4$ м. регистрации.

\section{5. Вариант широкополосного возбуждения}

Когда примеси, люминесцирующие в области $(0-0)$-линии БП, практически отсутствуют в объекте измерения, люминесценцию БП можно возбуждать и достаточно широким участком спектра ближнего УФ, к примеру лампой ДРШ-500 через фильтры УФС-2 и УФС-5. На рис. 5 показаны измерения аналитической линии БП в объектах нижнего конца концентрационного ряда при таком широкополосном возбуждении. Видно, что наряду с аналитической линией в спектре присутствует бесструктурное фоновое свечение с максимумом в районе $4100 \AA$ (не умещается в приведенный спектральный интервал). Это постороннее свечение принадлежит, по всей видимости, КР матрицы н-октана.

Благодаря тому, что барабанный объектодержатель фиксирует объекты в положении измерения достаточно надежно, можно значительно увеличить отношение сигнала к шуму (фону) в спектрах последних членов концентрационного ряда вычитанием сигнала, полученного от объекта с чистым можность, спектры дополнительно разделены по высоте вычитанием или сложением некоторого постоянного уровня. Видно, что таким способом можно измерить сигнал от объекта с концентрацией $10^{-12} \mu / \Omega$ (т. е. $\left.2,5 \cdot 10^{-13} 2 / M \Omega\right)$.

\section{6. Абсолютная чувствительность анализа}

Достигнутый предел чувствительности порядка $5 \cdot 10^{-13}$ 2/м вует $10^{9}$ молекулам БП в $1 \mathrm{~cm}^{3}$ - в принятом объеме объекта измерения. С другой стороны, понятно, что реально возбуждается и участвует в формировании сигнала лишь незначительная доля молекул БП полного объема объекта, который можно поэтому значительно умень- 

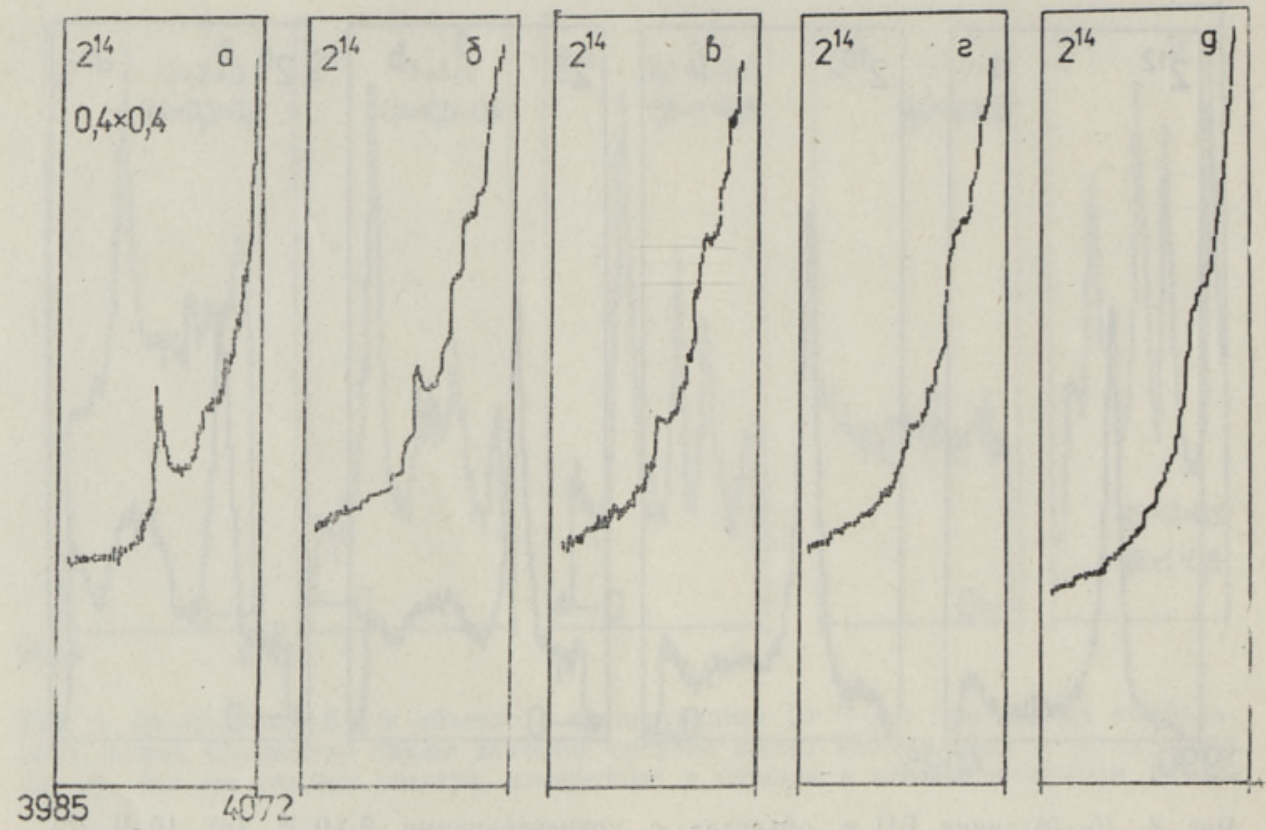

Рнс. 5. (0-0)-линия БП в объектах с концентрациями $3 \cdot 10^{-11}(a), 10^{-11}(6), 3 \cdot 10^{-12}$ $(в), 10^{-12} \mathrm{M} / \Omega($ (2) при широкополосном возбуждении. Свечение чистого н-октана $(\partial)$.

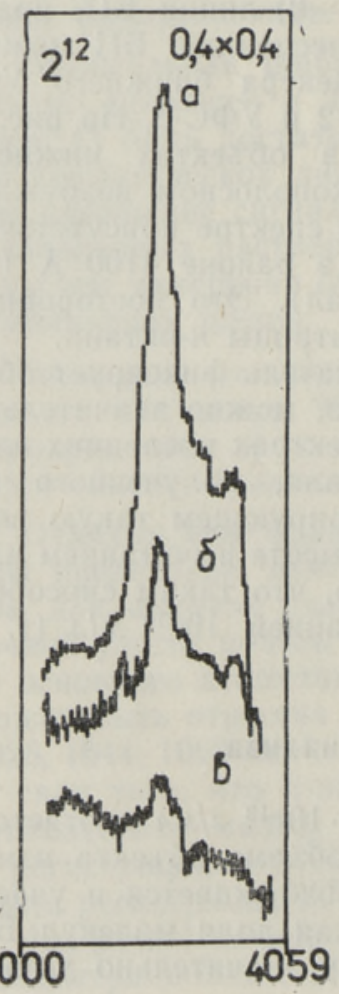

Рис. 6. (0-0)-линия БП в объектах с концентрациями $10^{-11}\left(\right.$ (a) $, 3 \cdot 10^{-12}(б), 10^{-12} \mathrm{M} / \Omega(в)$ при широкополосном возбуждении после вычитания фонового свечения.

шить, существенно повысив абсолютную чувствительность анализа. По-видимому, за счет некоторого дальнейшего уменьшения объема и увеличения интенсивности возбуждения можно продвинуться по пути повышения абсолютной чувствительности до величины порядка $10^{6}-10^{5}$ молекул БП. Мы сравнили раствор в ампуле с концентрацией $2 \cdot 10^{-9} 2 /$ с $^{3}$ и тот же раствор в капиллярной трубке в $0,005 \mathrm{~cm}^{3}$. Потеряв в сигнале в 4 раза, но выиграв в уменьшении объема в 200 раз, можно считать, что абсолютная чувствительность анализа в такой капиллярной трубке выше до двух порядков.

Самый перспективный способ повышения абсолютной чувствительности анализа связан с применением лазерного возбуждения. При этом важны три момента: 1) высокая селективность возбуждения; 2) возможность фокусировки энергии луча в малый объем, практически до 1 микрон $^{3}$, т, е. в $10^{12}$ раз меньше 
$1 \mathrm{~cm}^{3}$; 3) высокая интенсивность возбуждения, что позволяет значительно увеличивать количество фотонов флуоресценции, получаемых в единицу времени от каждой молекулы анализируемого соединения. В принципе, пределом чувствительности метода является одна молекула.

\section{7. Заключение}

В итоге проведенной работы можно считать, что чувствительность анализа БП увеличена на величину около двух порядков. На практике, при соблюдении перечисленых предосторожностей лабораторной работы и применении соответствующей измерительной техники, представляется возможным проводить анализы БП вплоть до концентраций $10^{-12} 2 / м л$. Для сравнения отметим, что такое количество БП содержится в среднем в $3 \bumpeq$ или в одном глотке чистого деревенского воздуха $\left[{ }^{16}\right]$.

Выражаю глубокую благодарность К. К. Ребане за постоянный интерес к работе.

\section{ЛИТЕРАТ УРА}

1. Muel, B., Lacrois, G. Caracterisation et dosage du 3,4 benzopyrène par spectrophotometrie de luminescence á $-190^{\circ} \mathrm{C}$. - Bull. Soc. Chim. France, 1960, N 11/12, p. 2139-2147.

2. Sawicki, E., Johns on, H. Thin-layer chromatographic characterization test for basic polynuclear compounds. Application to air pollution. - Mikrochim. Acta, 1964, N 2/4, p. 435-450; S a w icki, E., S tanley, S. W., Johns o n, H. Quenchofluorometric analysis for polynuclear compounds. - ibid., 1965 , N 1, p. $178-192$.

3. Sawicki, E. The separation and analysis of polynuclear aromatic hydrocarbons present in the human environment. - Chemist-analyst, 1964, v. 53 , p. $24-30$, $56-62,88-94$.

4. Ш а бад Л. М. Методы определения и изучения бластомогенности химических веществ. М., 1970, с. 206.

5. Т еплицк а я Т. А. Квазилинейчатые спектры люминесценции как метод иссле дования сложных природных органических примесей. М., 1971.

6. Sittig, M. Pollution Detection and Monitoring Handbook. Noyes Data Corp., Park Ridge. New Jersey - London, 1974, p. 331.

7. Ш польский Э. В. Линейные спектры флуоресценции органических соединений и их применения. - Успехи физ. наук, 1960, т. 71, вып. 2, с. 215-242; Проблемы происхождения и структуры квазилинейчатых спектров органических соединений при низких температурах. - Там же, 1962, т. 77, вып. 2, с. $321-$ 336; Новые данные о природе квазилинейчатых спектров органических соединений. - Там же, 1963 , т. 80 , вып. 2, с. $255-279$.

8. Р еб а е К. К. Элементарная теория колебательной структуры спектров примесных центров кристаллов. М., 1968.

9. Р еб а не К., С а а р и П., Т а м м Т. Природа «мультиплетов» и фононное крыло в спектрах некоторых систем Шпольского. - Изв. АН ЭССР. Физ. Матем., 1970 , т. 19 , № 2. с. $251-254$.

10. Персонов Р. И. Количественное определение 3,4-бензпирена по линейчатым спектрам флуоресценции при 77 К. - Ж. аналит. химии, 1962, т. 17, вып. 4, c. $506-510$.

11. Шаба д Л. М., Д икун П. П. Загрязнение атмосферного воздуха канцерогенным веществом 3,4-бензпиреном. Л., 1959.

12. Климова Л. А., Оглоблина А. И., Шпольский Э. В. К вопросу о природе зависимости резкости спектров ароматических углеводородов от их концентрации в замороженных Сер. физ., 1970 , т. 34 , вып. 6, с. $1361-1364$.

13. Отчет Института химии АН ЭССР по теме № ГП ЭССР 107:226 «Разработка методов получения чистых и особо чистых спектральных сольвентов», Таллин, 1975 . 
14. Т а мм Т. Б., С а ар и П. М. Соответствие компонентов мультиплетов спектров флуоресценции и фосфоресценции 1,2-бензпирена в твердых матрицах. Оптика и спектроскопия, 1975 , т. 38 , вып: 5 , с. $1029-1031$.

15. T a m m, T. On the drastic effects of inhomogeneity in Shpolsky system spectra. ENSV TA Toimet. Füüs. Matem., 1977, v. 26, N 1, p. $72-78$.

16. С те рин Х. Е., Ал ек санян В. Т., Жи жин Г. Н. Каталог спектров комбинационного рассеяния углеводородов. М., 1976.

\author{
Институт физики \\ Академии наук Эстонской ССР \\ Поступила в редакцию \\ $15 / \mathrm{V} 1980$
}

\title{
T. TAMM
}

\section{SPOLSKI MEETODIL PŌHINEVA BENSO(a)PUREENI ANALUUSI TUNDLIKKUSE SUURENDAMINE}

On kirjeldatud abinõude kompleksi, mis võimaldavad suurendada Špolski meetodil põhineva benso (a)püreeni analüüsi tundlikkust kuni $10^{-12}$ mooli/l. On analüüsitud metoodi kaht varianti - selektiivse ja mitteselektiivse ergutusega luminestsentsi - ning hinnatud analüüsi absoluutse tundlikkuse suurendamise viise.

\section{T. TAMM}

\section{IMPROVEMENT OF THE SENSITIVITY OF AN ANALYSIS OF BENZO(a)PYRENE BY THE SHPOLSKY METHOD}

The paper contains the description of a complex of measures for increasing the sensitivity of the analysis of benzo(a)pyrene by the Shpolsky method up to $10^{-12}$ moles per litre. Two modifications of the method, with selective and unselective excitation of luminescence, have been analyzed. The pathways for enhancing the absolute sensitivity of the analysis have been discussed. 